\title{
Los incidentalomas adrenales no funcionantes se asocian a una mayor incidencia de diabetes y pre-diabetes
}

Non-functioning adrenal incidentalomas are associated with an increased incidence of diabetes and pre-diabetes

\section{Objetivos}

Evaluar si los pacientes con incidentalomas adrenales no funcionantes (IANF) tienen mayor riesgo de desarrollar eventos cardiometabólicos que los que no los tienen.

\section{Diseño}

Estudio de cohorte llevado a cabo en EE.UU. Participaron 166 pacientes con IANF (grupo expuesto) y 740 sin tumor adrenal (no expuesto) con al menos tres años de seguimiento.

\section{Evaluación de factores pronósticos}

Fueron evaluados en forma retrospectiva adultos con el hallazgo incidental de tumores adrenales en imágenes abdominales. Fueron excluidos aquellos con diagnóstico documentado de patología suprarrenal (tumores funcionantes o histología diferente de adenoma benigno), patología hipofisaria y uso de corticoides sistémicos durante más de tres meses.
Lopez D y col. Ann Intern Med. 2016;165:533-542.

\section{Medición de resultados principales}

Fueron revisadas las historias clínicas desde el momento de la realización de la imagen para evaluar la aparición de hipertensión, trastornos del metabolismo de la glucosa -pre diabetes o diabetes tipo 2-, dislipemia, eventos cardiovasculares y enfermedad renal crónica, con un seguimiento promedio de 7,7 años. El análisis primario evaluó asociaciones independientes entre la exposición y los resultados incidentes usando modelos lineales generalizados ajustados, y el secundario, la relación entre los IANF y la fisiología del cortisol.

\section{Resultados principales}

Los pacientes con IANF tuvieron una incidencia más alta de trastornos del metabolismo de la glucosa que aquellos sin tumores adrenales. Ver tabla 1.

Tabla 1. Incidencia de trastornos del metabolismo de la glucosa en pacientes con incidentalomas adrenales no funcionantes.

\begin{tabular}{c|c|c|c} 
& Casos & Controles & Diferencia (IC 95\%) \\
\hline Trastornos del metabolismo de la glucosaa & $27,3 \%$ & $11,7 \%$ & $15,6 \%(6,9 \%$ a $24,3 \%)$ \\
\hline
\end{tabular}

Incluye pre-diabetes y diabetes tipo 2 .

No se observaron asociaciones significativas entre IANF y otros eventos cardiometabólicos. Los pacientes con mayor valor de cortisol post dexametasona, aunque dentro de los límites normales (menores a $1,8 \mathrm{mcg} / \mathrm{dL}$ ), presentaron mayor tamaño tumoral y mayor incidencia de diabetes.

\section{Conclusiones}

Los participantes con IANF tuvieron una incidencia significativa- mente mayor de trastornos del metabolismo de la glucosa que los del grupo control. Estos resultados muestran la necesidad de reevaluar los criterios de clasificación de los adenomas adrenales como "no funcionantes", considerando que existe un espectro continuo de secreción hormonal que ocasiona diferente riesgo metabólico.

Fuente de financiamiento: NIH (National Institutes of Health) y Fundación Caritativa Doris Duke.

\section{Comentario}

Debido al uso extendido y a la mayor resolución de los estudios por imágenes, el diagnóstico incidental de lesiones adrenales constituye un problema frecuente. El término "incidentaloma adrenal" abarca numerosas patologías que únicamente comparten su forma de diagnóstico. El desafío consiste en establecer si se trata de lesiones benignas o malignas, y si presentan o no hiperfunción hormonal. En particular, la evaluación del eje hipotálamo-hipofiso-adrenal presenta dificultad, debido a que puede sufrir interferencias por numerosos factores. Existe consenso en usar la prueba de supresión con $1 \mathrm{mg}$ de dexametasona (PSD$1 \mathrm{mg})$ como herramienta de pesquisa, para la que inicialmente se propuso un valor de corte de $5 \mu \mathrm{g} / \mathrm{dL}^{1}$. Sin embargo, dada la heterogeneidad y escasez de estudios prospectivos en este campo, existen ciertas controversias. Por ejemplo, las guías europeas publicadas en 2016 sugieren utilizar un valor de corte para la PSD-1mg de $1,8 \mu \mathrm{g} / \mathrm{dL}$, y que a los pacientes con valores inferiores no se les repitan determinaciones hormonales a menos que empeoren sus comorbilidades metabólicas ${ }^{2}$.

Si bien presenta limitaciones inherentes a su carácter observa- cional, luego de analizar los resultados del trabajo de Lopez y $\mathrm{col}$. que hemos resumido, es necesario considerar si estos adenomas son verdaderamente "no funcionantes", ya que, comparados con los pacientes sin tumores adrenales, estos pacientes presentaron casi el doble de riesgo de desarrollar algún trastorno del metabolismo de la glucemia, lo que invita a reevaluar si es necesario usar alguna otra determinación bioquímica u otro valor de corte para estratificar el riesgo metabólico dentro del continuo de secreción hormonal descripta en estos tumores.

\section{Conclusiones de la comentadora}

Son necesarios más estudios para establecer la relación entre los adenomas adrenales y la presencia de comorbilidades con impacto cardiovascular, y así definir criterios de diagnóstico, seguimiento y tratamiento adecuados. Mientras tanto, se recomienda un enfoque individualizado junto con un seguimiento interdisciplinario para realizar las intervenciones terapéuticas necesarias, ya sean farmacológicas o quirúrgicas.

Andrea Paissan [ Servicio de Endocrinología del Hospital Italiano de Buenos Aires. andrea.paissan@hospitalitaliano.org.ar ]

Paissan A. Los incidentalomas adrenales no funcionantes se asocian a una mayor incidencia de diabetes y pre-diabetes. Evid Act Pract Ambul. 2017; 20(4): 90. Comentado de: Lopez D, y col. "Nonfunctional" Adrenal Tumors and the Risk for Incident Diabetes and Cardiovascular Outcomes. Ann Intern Med. 2016;165:533-542.

Referencias bibliográficas

1 Grumbach M y col. Management of the Clinically Inapparent Adrenal Mass. Ann Intern Med. 2003;138: 424-429.

2 Fassnacht M y col. Management of adrenal incidentalomas: European Society of Endocrinology Clinical Practice Guideline. European Journal of Endocrinology (2016) 175, 2 Fassnacht 